\title{
A Case Study of Efficacy of Homoeopathic Medicines in Case of Infertility Due to Uterine Fibroid without any Surgical Procedure
}

\author{
Rahul Singh
}

Medical Advisor- Bhargava Phytolab Pvt. Ltd, Noida -201301 (U.P) India

\begin{abstract}
*Corresponding author
Dr. Rahul Singh, BHMS (Pune), HMD (London), CNCC (Delhi), Medical Advisor- Bhargava Phytolab Pvt. Ltd; Clinic: - Care 'N Cure Homoeopathic Health Centre 182, Main Road Mandawali, near badi Masjid, Delhi, India. E-mail: drsingh_29@rediff.com
\end{abstract}

Submitted: 07 Aug 2018; Accepted: 16 Aug 2018; Published: 08 Dec 2018

Citation: Rahul Singh. (2019). A Case Study of Efficacy of Homoeopathic Medicines in Case of Infertility Due to Uterine Fibroid without any Surgical Procedure. Med.Clin.Res, 4 (1), 1-5.

\begin{abstract}
This case study is about the efficacy of Homoeopathic medicines in treating the infertility caused due to Uterine Fibroid, which is a most common pelvic tumor in women. Day by day, case of uterine Fibroids are increasing, where they are causing difficulty in conceive or leading to miscarriages. Lower abdominal pain, irregular menstruation and abnormal uterine bleeding are other common symptoms. While conventional medicine does not offer any satisfactory treatment except surgical removal of Myomas (Myomectomy) or in extreme condition, complete removal of Uterus (hysterectomy), Homoeopathy is a good alternative for the treatment of Myomas.
\end{abstract}

At, Care 'N' Cure Homoeopathic Health center, Delhi, we have treated and still treating many cases of uterine fibroids by homoeopathic medicines for last more than 13 years. Out of them, around $70 \%$ have shown very good results. Most of them were referred for surgery by other doctors, but well selected homoeopathic medicine and proper counseling, leads the complete disappearance of fibroids. Duration of treatment varied from case to case depending on the size and total numbers of Myomas.

Outcome of our study on uterine fibroid is very encouraging. Some of the patients who were childless due to large and multiple uterine fibroid got conceived with the help of homoeopathy. These results are very encouraging and giving us the more confidence to work in the field of infertility.

\section{Introduction}

Uterine fibroids, also called as Leiomayomas or myomas, are very common benign (non-cancerous) over growths that develop in the muscular wall of the uterus. They can be very tiny to larger than a cricket ball in size. In most of the cases, there is more than one fibroid in the uterus. Sometimes fibroids can be asymptomatic, but most of the time due to their size and location, fibroids can lead to problems for some women like abdominal pain and heavy bleeding. Rarely can they develop in to cancer. Even though their pathogenesis is not clearly known, there is considerable evidence that estrogen and progesterone proliferates the growth of fibroids, as they rarely appear before menarche and regress after menopause.

\section{Background}

In the period of Hippocrates in 460-375 B.C., this lesion was known as the "uterine stone". Galen called this finding "scleromas" during the second century of the Christian period. The term fibroid was coined and introduced in 1860 by Rokitansky and in the 1863 by Klob. In 1854, a German pathologist named Virchow demonstrated that these neoplasms (fibroids) were composed from smooth muscle cells. It was Virchow who introduced the word "myoma".

Epidemiology of Uterine Fibroid

- Fibroids are the most common benign tumors in females.
- Fibroids are typically found during the reproductive years of females.

- Worldwide, $20-40 \%$ women will be diagnosed with fibroid (Obstetrics and Gynecology clinic,2000)

- The incidence is twice more common in black women than white.

- Prevalence rate is approx. 1 in 20 or 13.6 million women, globally.

- In India, $25 \%$ of women in their reproductive years have noticeable fibroids (National Institute of Health, India).

\section{Aetiology}

Fibroids originate from the thick wall of the uterus in different locations in and on the uterus and are categorized by where they grow:

\section{Types of Fibroids}

- Intramural Fibroids: These are the most common type of fibroid. These types appear within the lining of the uterus (endometrium). Intramural fibroids may grow larger and can stretch the womb.

- Subserosal Fibroids: Subserosal fibroids form on the outside of the uterus, which is called the serosa. They may grow large enough to make the womb appear bigger on one side. 
- Submucosal Fibroids: These types of tumors develop in the inner lining (myometrium) of the uterus. Submucosal tumors are not as common as other types, but when they do develop, they may cause heavy menstrual bleeding and trouble in conceiving.

- $\quad$ Pedunculated Fibroids: When subserosal tumors develop a stem (a slender base that supports the tumor), they become pedunculated fibroids.

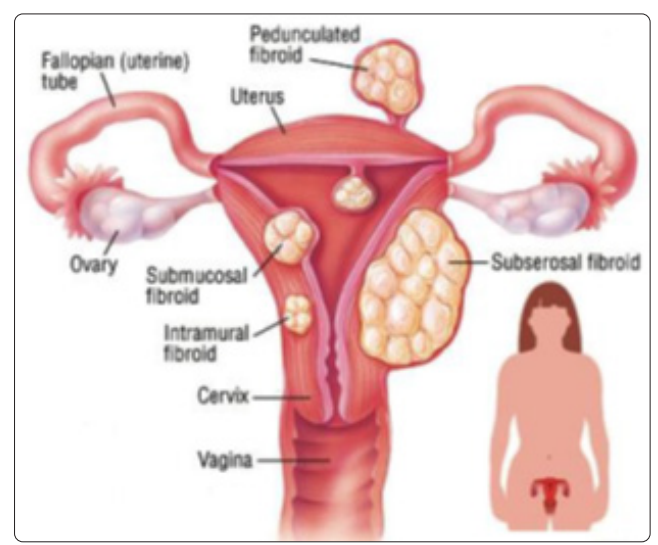

Risk factors

- $\quad$ Family history - Family history of fibroids increases the risk three times.

- $\quad$ Age - Fibroids become more common as women age. (mostly 30 to 50 years of age)

- $\quad$ Race - African-American are more prone to get Fibroid.

- Obesity - Overweight women are at higher risk for fibroids.

- Pregnancy - Due to an increased level of Estrogen.

Symptoms of Fibroids

It depends on the location and size of the tumors and number of tumors one have. If fibroid is very small, it may not cause any trouble. It can be shrink or disappears after menopause.

- $\quad$ Profuse bleeding during menstruation or Dysmenorrhea

- Pain and heaviness in the pelvis and lower abdomen

- Swelling or enlargement of the abdomen

- Lower backache

- Frequent urination

- $\quad$ Pain during sexual intercourse

- Irregular menstruation that lasts longer than usual

- Reproductive problems, such as infertility or repeated miscarriages

Investigations \& Diagnosis

Fibroids are most often found during a routine pelvic examination. This, along with an abdominal examination, may indicate a firm, irregular pelvic mass to the physician. In addition to a complete medical history and physical and pelvic and/or abdominal examination, a number of tests may show more information about fibroids:

- Trans-vaginal ultrasound (TVS)

- Magnetic resonance imaging (MRI)

- Hystero-salpingography- X-ray examination of the uterus and fallopian tubes that uses dye and is often performed to rule out tubal obstruction.

- Hysteroscopy- Visual examination of the canal of the cervix and the interior of the uterus using a viewing instrument (hysteroscope) inserted through the vagina.
- $\quad$ Laparoscopy - Using a slender device (the laparoscope), doctor see the inside of the abdomen. It is inserted through a small cut just below or through the navel. The doctor can see fibroids on the outside of the uterus with the laparoscope.

- Endometrial biopsy-A procedure in which a sample of tissue is obtained through a tube which is inserted into the uterus.

- $\quad$ Routine Blood test - To check for iron-deficiency anemia if heavy bleeding is caused by the tumor.

- $\quad$ Cat scan (CT) - Takes many X-ray pictures of the body from different angles for a more complete image.

Case study of infertility due to uterine fibroid

(Case 1)

Name: Mrs. S

Age: $32 / \mathrm{F}$

Occupation: Housewife

Registration No: 2912

Date of the first visit: $7^{\text {th }}$ October 2013

Chief Complaints:

1. Pain in lower abdomen and right side of the pelvis $<$ during menses.

2. Unable to conceive.

Associated Complaints:

Menses: Early and profuse with severe pain in the lower abdomen which goes to back.

$\Delta$ Feeling of excessive heaviness in uterine region. +++

$\Delta$ Leucorrhoea- yellowish discharge with itching at the vulva.

$\rightarrow$ Stool constipated most of the time

Past History:

$\rightarrow \quad$ Miscarriage in the $6^{\text {th }}$ month of pregnancy -2013

$\rightarrow$ Jaundice -2009

Family History: Nothing specific

Physical examination:

Respiratory rate: 13 breaths/minute

Abdomen: Swelling and tenderness in lower abdomen

Vitals:

Blood pressure: 110/74 $\mathrm{mmHg}$

Heart rate: 70 beats/min

Weight: $56 \mathrm{~kg}$

\section{Investigations}

1. First ultrasound (23 ${ }^{\text {rd }}$ August 2013) not attached -

Uterus: Fibroid of size $4.3 \mathrm{~cm} \mathrm{X} 3.0 \mathrm{~cm}$ in post myometrium.

Ovary: A cyst of about $1.7 \mathrm{~cm} \mathrm{X} 1.0 \mathrm{~cm}$ in Right Ovary.

On the basis of physical symptoms and investigations, secondary infertility due to uterine fibroid was diagnosed and homoeopathic treatment was started.

2. Second USG (TVS) on $5^{\text {th }}$ January 2014: Size of fibroid reduces to 3.1 X 2.8 X 2.1 (in cm) with a Nabothian cyst of 17 X $9 \mathrm{~mm}$ along with posterior lip. 


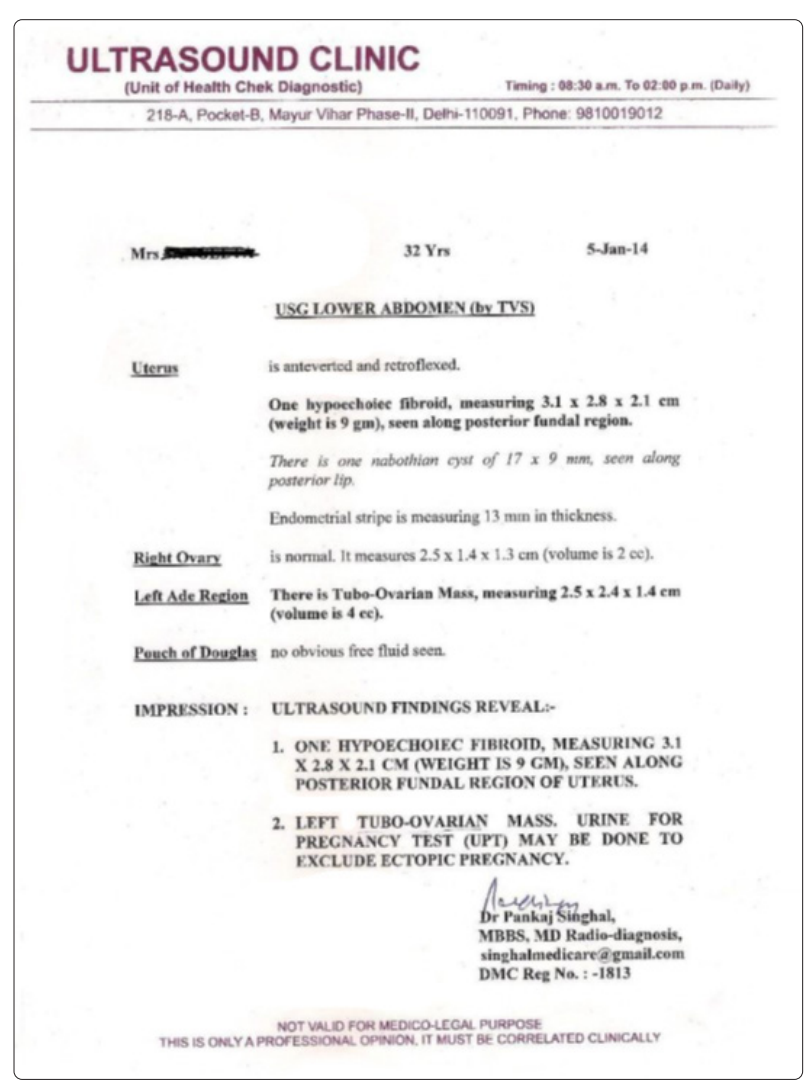

3. Third USG on 11th June 2014 confirms pregnancy of 5 weeks and reduced size of myoma $-28 \mathrm{~mm}$ (or $2.8 \mathrm{~cm}$ ).

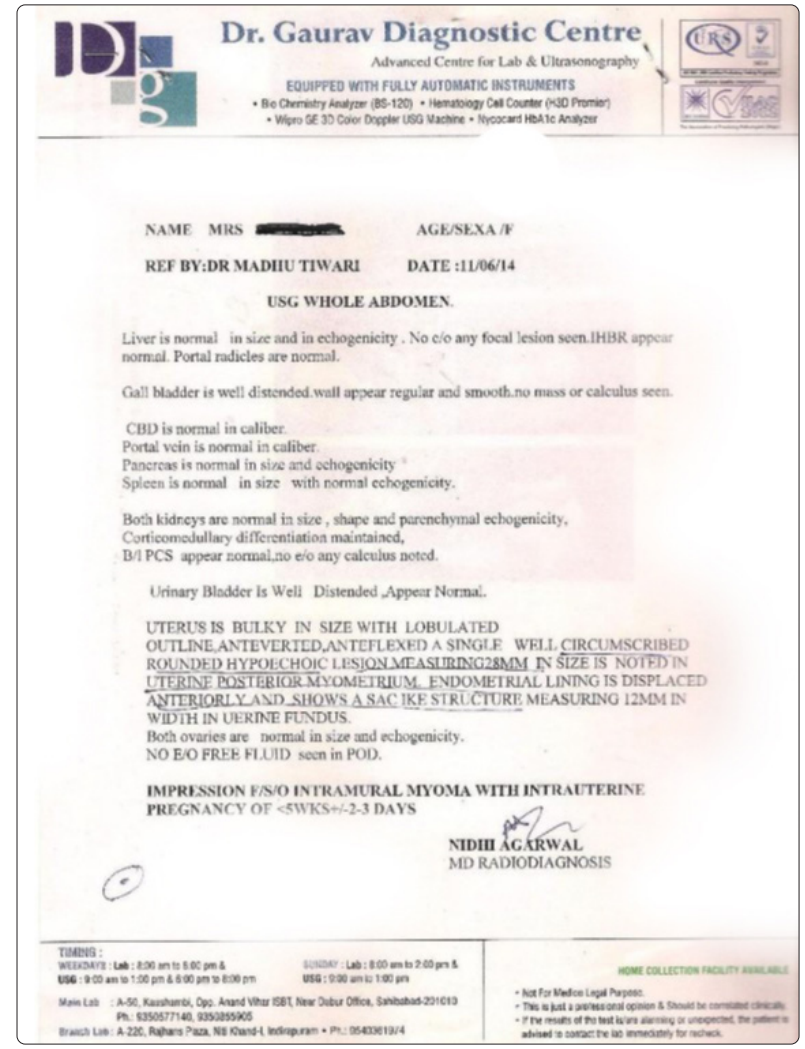

Now our prime concern was to save the Fetus and to carry a normal pregnancy.
4. The fourth USG on 4th December 2014 confirms the pregnancy of 30 weeks and with no sign of myoma/fibroid.

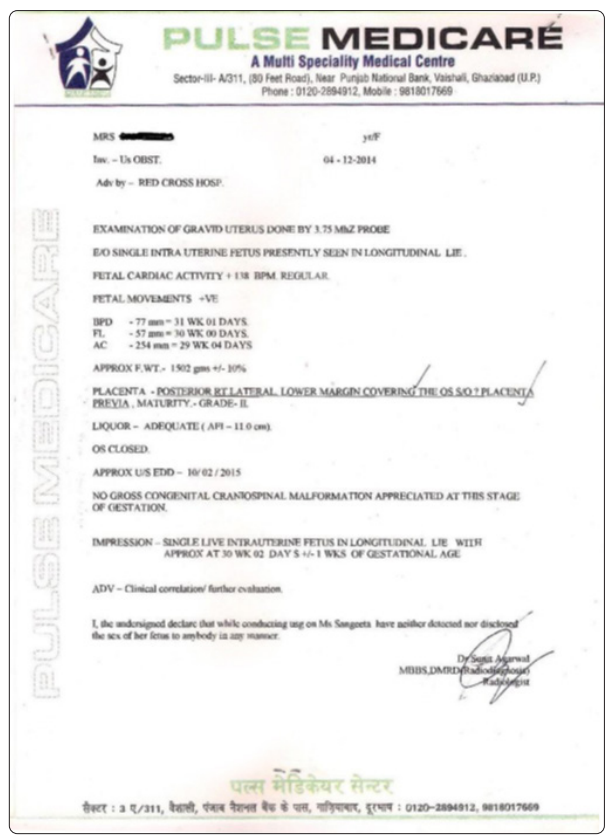

On $15^{\text {th }}$ February 2015: At full time, a normal and healthy baby boy was born (Weight: $2.5 \mathrm{Kg}$ ). According to her gynecologist, there was no any sign of Myoma.

Both mother and baby were normal and good in their health.

On 20 ${ }^{\text {th }}$ September 2015: Again she became pregnant for the second baby. Everything was normal.

$18^{\text {th }}$ April 2016: Ultrasound report suggests normal single intrauterine Gestation of 36 weeks, without any sign of Fibroid.

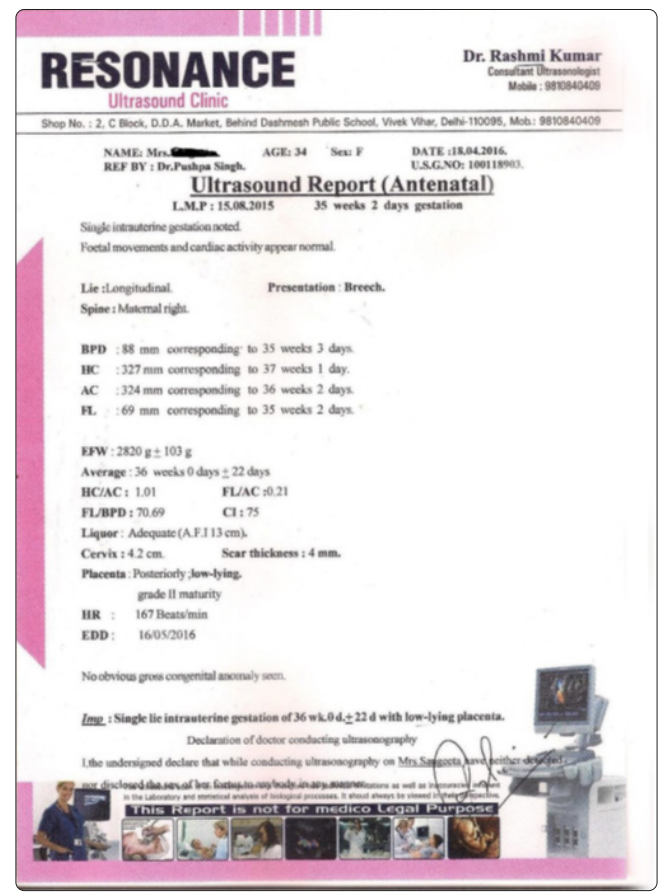

According to her gynecologist, the growth of baby was very good. There was no Fibroid. 


\section{Result}

15-05-2016: At full time, again a normal and healthy baby boy was born (this time Weight was: $3.10 \mathrm{Kg}$ ).

Now the couple was very happy, according to them they have completed their family. Previously they were praying for at least one child but now, by god grace, they have two children. They owing this blessing to god and to Homoeopathy.

Homoeopathic Medicines prescribed:

Sepia 30- tds

Thuja 1M, 10M - Single dose

Aurum mur. natronatum 3x - bd

Fraxinus Americana $Q \mathbf{~ - ~} 10$ drops tds

Case 2

Name: Mrs. K

Age: 28/F

Occupation: Housewife

Registration No: 3012

Date of the first visit: $2^{\text {nd }}$ February 2014

Chief Complaints:

1. Repeated miscarriages (3 times in two years).

Associated Complaints:

$\checkmark \quad$ Pain in the lower abdomen during menses.

Past History:

D 3 Miscarriages from 2012 to 2013

$\checkmark$ Chicken pox in childhood

Family History:

$\triangle$ Father hypertensive

Physical examination:

Respiratory rate: 14 breaths/minute

Abdomen: Soft and non-tender

Vitals:

Blood pressure: $120 / 80 \mathrm{mmHg}$

Heart rate: 74 beats/min

Weight: $54 \mathrm{~kg}$

Investigations \& Treatment

1. First USG (TVS) on $6^{\text {th }}$ February 2014 :

A fibroid of $1.8 \times 1.9 \mathrm{~cm}(18 \times 19 \mathrm{~mm})$ in the right lateral aspect of right adnexa.

A cyst of $3.4 \times 2.7 \mathrm{~cm}(34 \mathrm{X} 27 \mathrm{~mm})$ in the right ovary.

On the basis of investigations, her present complaints and her past history, secondary infertility due to uterine fibroid was diagnosed and homoeopathic treatment was started.

Result

After a regular follow up and proper homoeopathic treatment,

2. $2^{\text {nd }}$ USG was done on $10^{\text {th }}$ May 2014:

The USG report state that uterus and ovary were normal without any sign of cyst or fibroid.

After 2-3 months, she conceived and delivered a healthy baby at full term. Now couple have a 3 years old boy.

Homoeopathic Medicines prescribed:

Apis 30- tds

Thuja 1M - Single dose
Case 3

Name: Mrs. K. S

Age: $35 / \mathrm{F}$

Occupation: Housewife

Registration No: 4410

Date of the first visit: $19^{\text {th }}$ March 2017

Chief Complaints:

1. Multiple uterine fibroids

2. Inability to conceive (never conceived for last 11 years)

Associated Complaints:

D Hypothyroidism (stable as taking some allopathic medicine)

$>$ Irregular menstruation

Past History:

$\triangle$ Nothing specific

Family History:

D Mother - hypothyroidism

Physical examination:

Respiratory rate: 12 breaths/minute

Abdomen: Hard and tender

Vitals:

Blood pressure: $130 / 88 \mathrm{mmHg}$

Heart rate: 72 beats/min

Weight: $62 \mathrm{~kg}$

Investigations

1. Latest USG (TVS) before starting the homoeopathic treatment on 19th February 2017:

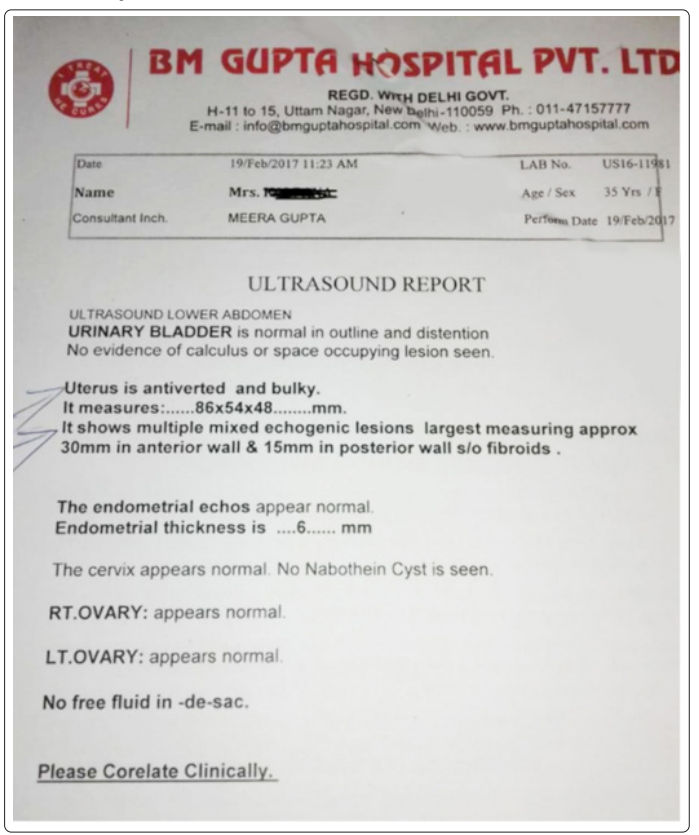

It shows multiple fibroids, largest measuring approx. $30 \mathrm{~mm}$ in anterior wall \& $15 \mathrm{~mm}$ in posterior wall of uterus.

Treatment

On the basis of investigations and patient history, 'primary infertility' due to uterine fibroid was diagnosed. Her gynecologist told them 
that natural pregnancy is difficult for them as husband is also having very low sperm count and both are aged, so, they should try IVF (in vitro fertilization).

But without removing fibroids, doctors are not ready to start IVF and couple do not want surgical removal of fibroids, so they came to us for homoeopathic treatment.

After complete analysis of the case, we have started homoeopathic treatment.

\section{Investigations}

2. $2^{\text {nd }}$ USG was done on 13th November 2017:

Shows few myomas, largest fibroid of $15 \mathrm{~mm}$ (previously of $30 \mathrm{~mm}$ ) in anterior wall of uterus and another of $12 \mathrm{~mm}$ in posterior wall.

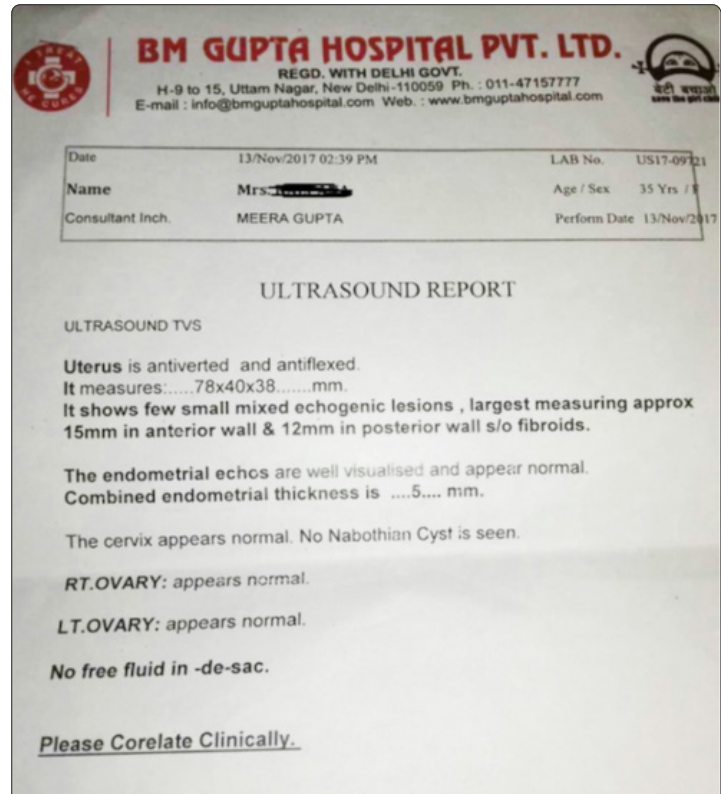

3. $3^{\text {rd }}$ USG was done on 6th May 2018:

Now a single fibroid of $1.5 \times 1.4 \mathrm{~cm}$ subserosal in posterior wall of uterus is remaining.

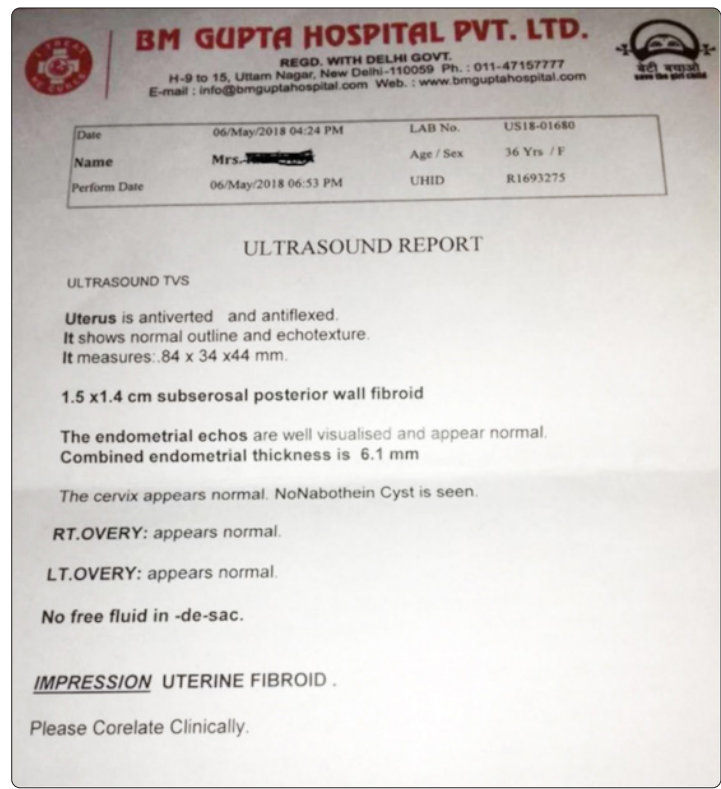

\section{Result}

Patient is still under our treatment. She is not taking any other medicines except homoeopathy. Her gynecologist is very surprised by the results of homoeopathic treatment. Now the couple is very optimistic and looking for IVF.

\section{Homoeopathic Medicines prescribed: \\ Natrum muraticum 30- tds \\ Thuja $1 \mathrm{M}$ - one dose, once in a month \\ Aurum mur. natronatum 3x - bd}

\section{Conclusion}

Cases of uterine fibroid are increasing globally. So, early detection of fibroid and their treatment is necessary to maintain the health of women. Treatment is required according to the size \& severity of fibroids. Conventional medicines do not offer a satisfactory treatment except for surgical removal of Myomas (Myomectomy) or in extreme condition, complete removal of Uterus (hysterectomy), Homoeopathy is a good alternative for the treatment of fibroid uterus. With homoeopathy, we can also avoid the complications of uterine fibroids and restore the health of the reproductive women [1-6].

\section{References}

1. V. G. Padubidri, Shirish N Daftary. Shaw's Textbook of Gynecology $16^{\text {th }}$ edition

2. Konar Hiralal (2013) Dutta DC Text Book of Obstetrics, $7^{\text {th }}$ edition, Jaypee Brothers Medical Publisher.

3. J T Kent (1998) Repertory of the Homoeopathic Materia Medica by, B. Jain Publishers, Reprint edition.

4. William Boericke (2010) New Manual of Homoeopathic Materia Medica \& Repertory by, MD, B. Jain publishers.

5. Bruce, Debra F (1998) "Fibroids." In The Unofficial Guide to Alternative Medicine. New York, NY: McMillan General Reference 278-281.

6. Naik Shubash, Nathan L.T (2006) "Indian prevalence rate, Fibroid uterus, National Institute of Health Science 275-278.
Copyright: (02018 Rahul Singh. This is an open-access article distributed under the terms of the Creative Commons Attribution License, which permits unrestricted use, distribution, and reproduction in any medium, provided the original author and source are credited. 